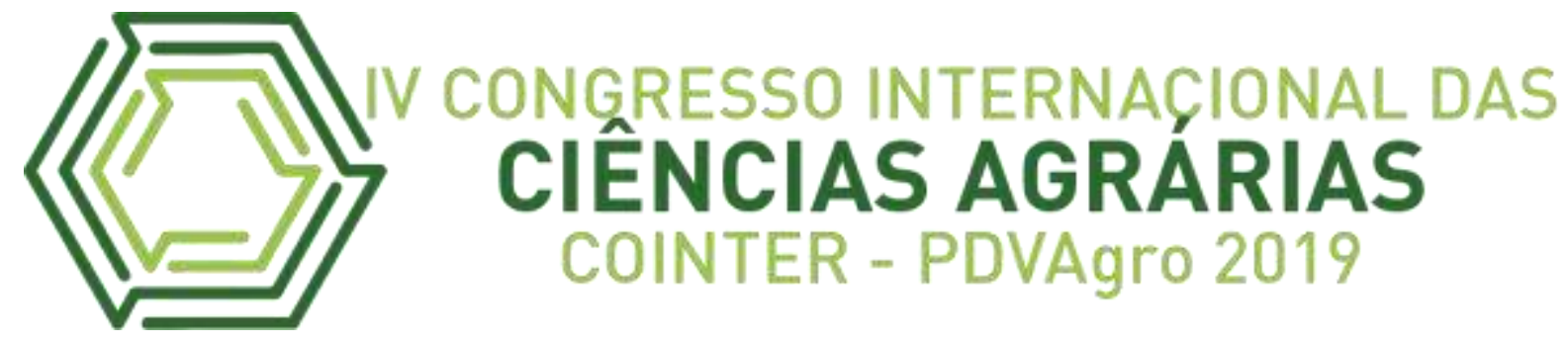

\author{
COMPOSIÇÃO FLORÍSTICA E FITOSSOCIOLÓGIA EM UMA ÁREA DE \\ MANEJO FLORESTAL NA FLORESTA NACIONAL DO TAPAJÓS, BELTERRA \\ PARÁ
}

\author{
FLORISTIC COMPOSITION AND PHYTOSOCIOLOGY IN A FOREST \\ MANAGEMENT AREA IN TAPAJÓS NATIONAL FOREST, BELTERRA PARÁ
}

COMPOSICIÓN FLORÍSTICA Y FITOSOCIOLOGÍA EN UN ÁREA DE MANEJO
FORESTAL EN EL BOSQUE NACIONAL DE TAPAJÓS, BELTERRA PARÁ

Apresentação: Comunicação Oral

Hanna Kassia Machado da Silva ${ }^{1}$; Osvaldo Júnior Moraes Moreira ${ }^{2}$; Ana Kaira Canté da Conceição ${ }^{3}$; José Humberto Fernandes da Rocha Sobrinho ${ }^{4}$; Nilton Junior Lopes Rascon ${ }^{5}$

\title{
DOI: https://doi.org/10.31692/2526-7701.IVCOINTERPDVAgro.2019.0154
}

\section{Resumo}

No decorrer dos anos foi atribuído grande importância para as florestas tropicais, principalmente pelos seus recursos naturais, sociais e econômicos, devido o fato de serem encontradas distintas regiões de vegetação. O objetivo do presente trabalho foi estudar a estrutura horizontal, distribuição espacial e composição florística na Floresta Nacional do Tapajós em uma área de manejo florestal, no município de Belterra Pará. Este estudo foi realizado na Área de Manejo Florestal (AMF) Anambé, situada na Floresta Nacional do Tapajós. O clima da região é do tipo Ami, segundo a classificação de Köppen, com temperatura em média de $25,5^{\circ} \mathrm{C}$ anual. Os dados deste estudo foram provenientes de um inventário 100\% realizado pela COOMFLONA (Cooperativa Mista da Flona do Tapajós). A diversidade foi calculada mediante o emprego do índice de Shannon (H'), e a uniformidade através da equabilidade de Pielou $(\mathrm{J})$. Em relação à estrutura horizontal, foram considerados os seguintes parâmetros para cada espécie: Densidade Absoluta (DA) e Densidade Relativa (DR); Dominância Absoluta (DoA) e Dominância Relativa (DoR); Frequência Absoluta (FA), Frequência Relativa (FR) e Índice Valor de Importância (IVI). E para a distribuição espacial o índice de Payandeh (1970). A área de estudo apresentou um tamanho de 1.723,70 ha, onde foram identificados 20.561 indivíduos (11,23 ind.ha $\left.^{-1}\right)$ distribuídos em 31 famílias e 100 espécies diferentes. As famílias botânicas que apresentaram maior número de indivíduos foram Sapotaceae (5.624), Fabaceae (4.841) e Lecythidaceae (3.708). Em relação à riqueza de espécies, as famílias com maior representatividade foram Fabaceae (37), Lauraceae (7), Moraceae (5) e Sapotaceae (5). As espécies mais abundantes na área, em ordem decrescente,

\footnotetext{
${ }^{1}$ Engenharia Florestal, Universidade Federal do Oeste do Pará, rhanna_ptra@hotmail.coml

2 Engenharia Florestal, Universidade Federal do Oeste do Pará, jumoreira3133@gmail.com

${ }^{3}$ Engenharia Florestal, Universidade Federal do Oeste do Pará, anakaira17@gmail.com

${ }^{4}$ Engenharia Florestal, Universidade Federal do Oeste do Pará, humbertofrs18@gmail.com

${ }^{5}$ Analista Ambiental, Instituto Chico Mendes de Conservação da Biodiversidade,nilton.rascon@icmbio.gov.br
} 
foram: Manilkara huberi (Ducke) A. Chev (maçaranduba), Couratari guianensis Aubl (Tauari) e Pouteria bilocularis (H.Winkl.) Baehni (goiabão),. Nesse contexto, a área apresentou um Índice de Shannon - Wiener (H') de 3,65 e equabilidade de Pielou (J) de 0,79. A área em estudo apresentou um crescente número de indivíduos nos centros de classe de diâmetro (CCDAP) de $45 \mathrm{~cm}$ a $95 \mathrm{~cm}$, em relação ao volume a área apresentou 69, $54 \mathrm{~m}^{3} \cdot \mathrm{ha}^{-1}$, a distribuição espacial pelo índice de Payandeh (Pi) constatou $84 \%$ das espécies com distribuição agregada, $10 \%$ tendência a agrupamento e $6 \%$ aleatória. A partir desses dados podemos inferir que a área apresenta alta diversidade de espécies e bem distribuídas. Além disso, apresenta estoque para potencial exploração florestal.

Palavras-Chave: Estrutura horizontal; Amazônia; Unidade de Conservação

\section{Resumen}

A lo largo de los años, se ha otorgado gran importancia a los bosques tropicales, principalmente por sus recursos naturales, sociales y económicos, debido al hecho de que se encuentran distintas regiones de vegetación. El objetivo del presente trabajo fue estudiar la estructura horizontal, la distribución espacial y la composición florística en el Bosque Nacional Tapajós en un área de manejo forestal en el municipio de Belterra Pará. Este estudio se realizó en el Área de Manejo Forestal de Amambé (AMF) Bosque Nacional Tapajós. El clima de la región es del tipo Ami, según la clasificación de Köppen, con una temperatura media anual de $25,5^{\circ} \mathrm{C}$. Los datos de este estudio provienen de un inventario $100 \%$ realizado por COOMFLONA (Cooperativa Mixta Flona de Tapajós). La diversidad se calculó utilizando el índice de Shannon ( $\left.\mathrm{H}^{\prime}\right)$, y la uniformidad a través de la igualdad de Pielou (J). Con respecto a la estructura horizontal, se consideraron los siguientes parámetros para cada especie: Densidad absoluta (AD) y Densidad relativa (DR); Dominio absoluto (DoA) y Dominio relativo (DoR); Frecuencia absoluta (FA), frecuencia relativa (RF) e índice de importancia del valor (IVI). Y para la distribución espacial del índice de Payandeh (1970). El área de estudio tenía un tamaño de 1,723.70 ha, que identificó 20,561 individuos $(11,23$ ind.ha-1) distribuidos en 31 familias y 100 especies diferentes. Las familias botánicas que presentaron el mayor número de individuos fueron Sapotaceae (5.624), Fabaceae (4.841) y Lecythidaceae (3.708). En cuanto a la riqueza de especies, las familias con mayor representación fueron Fabaceae (37), Lauraceae (7), Moraceae (5) y Sapotaceae (5). Las especies más abundantes en el área, en orden descendente, fueron: Manilkara huberi (Ducke) A. Chev (maçaranduba), Couratari guianensis Aubl (Tauari) y Pouteria bilocularis (H.Winkl.) Baehni (guayaba). En este contexto, el área presentó un índice de Shannon - Wiener (H ') de 3.65 y la igualdad de Pielou (J) de 0.79. El área en estudio presentó un número creciente de individuos en los centros de clase de diámetro (CCDAP) de $45 \mathrm{~cm}$ a $95 \mathrm{~cm}$, en relación con el volumen que el área presentó $69,54 \mathrm{~m}^{3} \cdot \mathrm{ha}^{-1}$, de acuerdo con la distribución espacial por el índice de Payandeh (Pi) $84 \%$ de las especies presentan distribución agregada, $10 \%$ de tendencia a agrupamiento y $6 \%$ al azar. De estos datos podemos inferir que el área tiene una gran diversidad de especies y está bien distribuida. Además, tiene stock para la explotación forestal potencial.

Palabras Clave: Horizontal structure; Amazon; Conservation Unit 


\begin{abstract}
Over the years, great importance has been attached to tropical forests, especially for their natural, social and economic resources, due to the fact that they are considered distinct regions of vegetation. The objective of the present work was to study the horizontal structure, spatial distribution and forest composition in the Tapajós National Forest in a forest management area in the municipality of Belterra Pará. This study was carried out in the Amambé Forest Management Area (AMF). Tapajós National Forest. The climate of the region is Ami, according to the Köppen classification, with an average annual temperature of $25.5^{\circ} \mathrm{C}$. The data from this study came from a $100 \%$ inventory conducted by COOMFLONA (Flona de Tapajós Mixed Cooperative). Diversity was calculated using Shannon index (H '), and uniformity through Pielou (J) equability. Regarding the horizontal structure, the following parameters were considered for each species: Absolute Density (AD) and Relative Density (DR); Absolute Dominance (DoA) and Relative Dominance (DoR); Absolute Frequency (FA), Relative Frequency (RF) and Value Importance Index (IVI). For spatial distribution the Payandeh index (1970). The study area had a size of 1,723.70 ha, which identified 20,561 individuals $\left(11,23\right.$ ind.ha $\left.^{-1}\right)$ distributed in 31 families and 100 different species. The botanical families that presented the largest number of individuals were Sapotaceae $(5,624)$, Fabaceae $(4,841)$ and Lecythidaceae $(3,708)$. Regarding species richness, the families with the largest representation were Fabaceae (37), Lauraceae (7), Moraceae (5) and Sapotaceae (5). The most abundant species in the area, in descending order, were: Manilkara huberi (Ducke) A. Chev (maçaranduba), Couratari guianensis Aubl (Tauari) and Pouteria bilocularis (H.Winkl.) Baehni (guava). In this context, the area presented a Shannon - Wiener Index (H ') of 3.65 and Pielou $(\mathrm{J})$ equability of 0.79 . The study area showed a growing number of individuals in diameter class centers (CCDAP) from $45 \mathrm{~cm}$ to $95 \mathrm{~cm}$, not following the "inverted j" pattern common in unequal forests. Regarding volume, the area presented $69,54 \mathrm{~m}^{3} \cdot \mathrm{ha}^{-1}$, according to the spatial distribution by the Payandeh (Pi) index. $84 \%$ of the species presented aggregate distribution, $10 \%$ clustering tendency and $6 \%$ random. From these data we can infer that the area has high species diversity and well distributed. In addition, it has stock for potential forest exploitation.
\end{abstract}

Keywords: Estructura horizontal; Amazonas; Unidad de Conservacion

\title{
Introdução
}

No decorrer dos anos foi atribuído grande importância para as florestas tropicais, principalmente pelos seus recursos naturais, sociais e econômicos, devido o fato de serem encontradas distintas regiões de vegetação (Lima et al.,2012). A Floresta Nacional do Tapajós é uma unidade de conservação criada em 1974, sendo muito estudada na parte de ciências florestais, no entanto ainda há necessidade de se estudar as potencialidades florestais dessa área (IBAMA, 2004; Gonçalves e Santos, 2008; Espirito Santo et al., 2008).

O estudo sobre a composição, estrutura e dinâmica dos ecossistemas florestais, são importantes para conservação e preservação da flora, além de contribuir nas praticas de 
manejo. Contudo, o conhecimento sobre a estrutura da floresta são informações fundamentais para a aplicação na produção sustentável (Lima et al., 2012). Nesse contexto, conhecer e estudar a estrutura da floresta é importante para a análise dos recursos florestais, sendo também fundamental para os critérios de colheita do plano de manejo florestal, avaliar o desenvolvimento da floresta e realizar tratamentos silviculturais (Gama et al., 2005 e Gama et al., 2007).

A distribuição espacial também é de suma importância para o estudo da floresta, podendo ser afetada por um conjunto de fatores sendo eles: bióticos e abióticos, ecologia da espécie, crescimento entre outros, no qual são importantes para estudos de regeneração natural, competição e dispersão da espécie (Lehn e Resende, 2007). Além disso, a floresta Amazônica em relação à composição florística ainda é pouco conhecida, dificultando o mapeamento da distribuição das plantas e da biodiversidade da região, sendo que esse estudo pode contribuir no planejamento para a conservação e uso sustentável do bioma (Hopkins 2007).

O objetivo do presente trabalho foi estudar a estrutura horizontal, distribuição espacial e composição florística na Floresta Nacional do Tapajós em uma área de manejo florestal, no município de Belterra Pará.

\section{Fundamentação Teórica}

O estudo das florestas sempre foi uma questão importante para o mundo, porem nós últimos anos que as florestas tiveram sua devida importância, principalmente depois das mudanças climáticas sofridas por ações antrópicas. Diante disso, os estudos sobre a composição florística são uteis para a interpretação do comportamento de comunidades florestais, servindo de base para projetos de conservação e preservação da mesma (Cubas, 2011).

As mudanças que veem correndo na Amazônia necessitam de um melhor aproveitamento socioeconômico, principalmente para a manutenção da biodiversidade. Com isso, podemos enfatizar a importância dos estudos florísticos e fitossociológico são informações qualitativas e quantitativas importantes para o conhecimento das espécies e habitats (Lima Filho et al., 2004 e Oliveira et al., 2008). 


\section{Metodologia}

\section{Área de estudo}

Este estudo foi realizado na Área de Manejo Florestal (AMF) Anambé, situada na Floresta Nacional do Tapajós, próximo ao km 117 da BR 163 (Santarém/Cuiabá). Os dados foram obtidos na Unidade de Produção Anual (UPA) 12, situada entre os paralelos $2^{\circ} 45^{\prime}$ e $4^{\circ} 10^{\prime} \mathrm{S}$ e os meridianos $54^{\circ} 45^{\prime}$ e $55^{\circ} 30^{\prime} \mathrm{W}$, o acesso foi feito através da BR 163.

De acordo com o IBAMA (2004) O clima da região é do tipo Ami, segundo a classificação de Köppen, com temperatura em média de $25,5{ }^{\circ} \mathrm{C}$ anual, com maiores precipitações nos meses de janeiro a maio, a região é caracterizada por dois a três meses de seca por ano e uma precipitação em média de $1.820 \mathrm{~mm}$. O tipo de solo predominante na área é o Latossolo amarelo distrófico. Segundo Veloso (1991) a vegetação é classificada como floresta ombrófila densa, caracterizado por arvores de grande porte.

\section{Coleta de dados}

Os dados deste estudo foram provenientes de um inventário $100 \%$ realizado pela COOMFLONA (Cooperativa Mista da FLONA do Tapajós) na UPA (Unidade de Produção Anual) 12 cuja área totalizou 1723,7 há. Foram inventariadas espécies comerciais ou potenciais ao comércio, sendo o nível de inclusão de $35 \mathrm{~cm}$ de DAP (Diâmetro Altura do Peito), teve sua circunferência à altura do peito medida a $1,30 \mathrm{~m}$ do solo e a altura comercial mensurada. As espécies foram identificadas in loco por parabotânicos e posteriormente foram tabuladas.

\section{Analise dos dados}

A diversidade foi calculada mediante o emprego do índice de Shannon (H'), na base logarítmica natural, e a uniformidade através da equabilidade de Pielou (J), conforme Magurran (1988).

Em relação à estrutura horizontal, conforme recomendado por Müller- Dombois e Ellemberg (1974) foram considerados os seguintes parâmetros para cada espécie: Densidade Absoluta (DA) e Densidade Relativa (DR); Dominância Absoluta (DoA) e Dominância Relativa (DoR); Frequência Absoluta (FA), Frequência Relativa (FR) e Valor de Importância 
(VI). A partir da soma dos valores relativos dessas três estimativas foi calculado o índice do valor de importância (IVI), introduzido por Curtis \& McIntosh (1951).

A estrutura diamétrica foi analisada distribuindo-se as árvores em classes de DAP de $10 \mathrm{~cm}$. Para analisar o incremento periódico entre as medições usou-se intervalo de classe de diâmetro de $10 \mathrm{~cm}$, consideraram-se todas as árvores vivas observadas na área (Gonçalves e Santos, 2008).

Por sua vez, o índice de Payandeh (1970), foi calculado com base na razão entre a variância do número de árvores por parcela e a média do número de árvores:

$$
P_{i}=\frac{S_{i}^{2}}{M_{i}} \quad M_{i}=\frac{\sum_{j=1}^{J} n_{i j}}{u T} \quad S_{i}^{2}=\frac{\sum_{j=1}^{J} n_{i j}^{2}-\frac{\left(\sum_{i=1}^{J} n_{i j}\right)^{2}}{u_{T}}}{u_{T}-1}
$$

Em que:

Si2 = variância do número de árvores da i-ésima espécie;

Mi = média do número de árvores da i-ésima espécie;

nij = número de árvores da i-ésima espécie na j-ésima parcela;

uT = número total de parcelas amostradas; e

$\mathrm{j}=1,2, \ldots, \mathrm{UT}$.

De acordo com os possiveis resultados obtidos para este índice, considera-se que: se Pi $<1,0$ a espécie apresentou padrão de distribuição espacial do tipo aleatório; se $1 \leq \mathrm{Pi} \leq 1,5$, tendência ao agrupamento e; se $\mathrm{Pi}>1,5$, agrupamento ou agregado.

\section{Resultados e Discussão}

A área de estudo apresentou um tamanho de 1.723,70 ha, onde foram identificados 20.561 indivíduos (11,23 ind.ha $\left.{ }^{-1}\right)$ distribuídos em 31 famílias e 100 espécies diferentes (Tabela 1). As famílias botânicas que apresentaram maior número de indivíduos foram Sapotaceae (5.624), Fabaceae (4.841) e Lecythidaceae (3.708). Em relação à riqueza de 
espécies, as famílias com maior representatividade foram Fabaceae (37), Lauraceae (7), Moraceae (5) e Sapotaceae (5).

Resultados semelhantes foram estudados por Condé e Tonini (2013) em uma floresta ombrófila densa na Amazônia, com um total de 4.724 indivíduos (525 ind.ha ${ }^{-1}$ ) constatando as mesmas famílias com maior frequência e riqueza de espécies. Com isso, o número de indivíduos e riqueza são fatores importantes para supremacia e perpetuação destas famílias, além de ser responsáveis por caracterizar a floresta (Silva et al., 2014).

Gonçalves e Santos (2008) em uma mesma área na Floresta Nacional do Tapajós, encontrou 8 famílias que contribuíram com mais de 50\% do total de espécies e 30\% das famílias contribuíram com apenas uma espécie. Além disso, a abundância de indivíduos nem sempre é proporcional ao número de espécies, ou seja, poucas espécies podem ser representadas por grandes populações ou uma única espécie pode ser muito abundante na comunidade. Essa observação é extensiva também, às famílias, que podem apresentar alta riqueza, mas serem pouco abundantes (Silva et al., 2014).

Tabela 1. Lista de espécies de acordo com os valores de densidade absoluta, densidade relativa, frequência absoluta, frequência relativa; dominância absoluta, frequência relativa e índice de valor de importância, na Floresta Nacional do Tapajós. Fonte: autor próprio

\begin{tabular}{|c|c|c|c|c|c|c|c|}
\hline Espécie/Família & DA & DR & FA & FR & DoA & DoR & IVI \\
\hline Anacardiaceae & 0,19 & 1,60 & 50,32 & 2,52 & $\mathbf{0 , 0 7}$ & 1,47 & 1,86 \\
\hline Anacardium spruceanum Benth. ex Engl. & 0,04 & 0,33 & 12,31 & 0,62 & 0,02 & 0,38 & 0,44 \\
\hline Astronium lecointei Ducke & 0,15 & 1,27 & 38,00 & 1,90 & 0,06 & 1,10 & 1,42 \\
\hline Apocynaceae & 0,14 & 1,17 & 28,45 & 1,42 & 0,04 & 0,78 & 1,12 \\
\hline Aspidosperma album (Vahl) Ben. ex Pichon. & 0,03 & 0,28 & 11,25 & 0,56 & 0,01 & 0,21 & 0,35 \\
\hline Aspidosperma auriculatum Markgr. & 0,02 & 0,16 & 5,31 & 0,27 & 0,01 & 0,24 & 0,22 \\
\hline Geissospermum sericeum Benth. \& Hook. f. ex Miers & 0,09 & 0,73 & 11,89 & 0,59 & 0,02 & 0,32 & 0,55 \\
\hline Araliaceae & $\mathbf{0 , 0 1}$ & $\mathbf{0 , 1 0}$ & 4,46 & 0,22 & $\mathbf{0 , 0 0}$ & 0,08 & 0,14 \\
\hline Schefflera morototoni (Aubl.) Maguire, Steyerm. \& Frodin & 0,01 & 0,10 & 4,46 & 0,22 & 0,00 & 0,08 & 0,14 \\
\hline Bignoniaceae & 0,24 & 2,02 & 63,27 & 3,17 & 0,12 & 2,34 & 2,51 \\
\hline Handroanthus serratifolius (Vahl) S.O. Grose & 0,10 & 0,84 & 27,18 & 1,36 & 0,04 & 0,71 & 0,97 \\
\hline Jacaranda copaia (Aubl.) D. Don & 0,02 & 0,17 & 6,58 & 0,33 & 0,01 & 0,10 & 0,20 \\
\hline Tabebuia incana A.Gentry & 0,12 & 1,01 & 29,51 & 1,48 & 0,08 & 1,52 & 1,34 \\
\hline Boraginaceae & $\mathbf{0 , 0 5}$ & 0,45 & 14,44 & 0,72 & 0,02 & $\mathbf{0 , 3 1}$ & 0,49 \\
\hline Cordia goeldiana Huber & 0,05 & 0,45 & 14,44 & 0,72 & 0,02 & 0,31 & 0,49 \\
\hline Burseraceae & 0,09 & 0,79 & 28,24 & 1,41 & 0,04 & 0,88 & 1,03 \\
\hline Protium paniculatum Engl. & 0,00 & 0,04 & 1,27 & 0,06 & 0,00 & 0,04 & 0,05 \\
\hline Protium polybotryum (Turcz.) Engl. & 0,08 & 0,64 & 22,51 & 1,13 & 0,04 & 0,79 & 0,85 \\
\hline Protium puncticulatum J.F.Macbr. & 0,00 & 0,00 & 0,21 & 0,01 & 0,00 & 0,00 & 0,01 \\
\hline Trattinnickia rhoifolia Willd. & 0,01 & 0,11 & 4,25 & 0,21 & 0,00 & 0,04 & 0,12 \\
\hline
\end{tabular}




\begin{tabular}{|c|c|c|c|c|c|c|c|}
\hline Calophyllaceae & 0,00 & 0,02 & 1,06 & 0,05 & 0,00 & 0,02 & $\mathbf{0 , 0 3}$ \\
\hline Caraipa richardiana Cambess. & 0,00 & 0,02 & 1,06 & 0,05 & 0,00 & 0,02 & 0,03 \\
\hline Caryocaraceae & 0,44 & 3,66 & 90,66 & 4,54 & 0,34 & 6,68 & 4,96 \\
\hline Caryocar glabrum (Aubl.) Pers. & 0,28 & 2,32 & 47,98 & 2,40 & 0,21 & 4,25 & 2,99 \\
\hline Caryocar villosum (Aubl.) Pers. & 0,16 & 1,34 & 42,68 & 2,13 & 0,12 & 2,43 & 1,97 \\
\hline Clusiaceae & 0,02 & 0,18 & 7,43 & 0,37 & 0,01 & 0,12 & 0,22 \\
\hline Symphonia globulifera L.f. & 0,02 & 0,18 & 7,43 & 0,37 & 0,01 & 0,12 & 0,22 \\
\hline Combretaceae & 0,13 & 1,10 & 34,61 & 1,73 & 0,05 & 1,01 & 1,28 \\
\hline Terminalia dichotoma G. Mey. & 0,13 & 1,10 & 34,61 & 1,73 & 0,05 & 1,01 & 1,28 \\
\hline Euphorbiaceae & 0,09 & 0,75 & 26,11 & 1,31 & 0,03 & 0,51 & 0,86 \\
\hline Glycydendron amazonicum Ducke & 0,01 & 0,06 & 2,76 & 0,14 & 0,00 & 0,06 & 0,09 \\
\hline Hevea brasiliensis (Willd. ex A. Juss.) Müll. Arg. & 0,06 & 0,47 & 15,07 & 0,75 & 0,01 & 0,28 & 0,50 \\
\hline Joannesia heveoides Ducke & 0,03 & 0,22 & 8,28 & 0,41 & 0,01 & 0,17 & 0,27 \\
\hline Fabaceae & 2,81 & 23,54 & 672,40 & 33,64 & 1,30 & 25,87 & 27,69 \\
\hline Albizia duckeana L. Rico & 0,04 & 0,36 & 14,01 & 0,70 & 0,01 & 0,29 & 0,45 \\
\hline Alexa grandiflora Ducke & 0,23 & 1,94 & 48,20 & 2,41 & 0,10 & 2,06 & 2,14 \\
\hline Apuleia molaris Spruce ex Benth. & 0,16 & 1,34 & 35,46 & 1,77 & 0,08 & 1,55 & 1,55 \\
\hline Bowdichia nitida Spruce ex Benth. & 0,03 & 0,26 & 10,40 & 0,52 & 0,01 & 0,19 & 0,32 \\
\hline Cedrelinga cateniformis (Ducke) Ducke & 0,07 & 0,60 & 20,38 & 1,02 & 0,06 & 1,12 & 0,91 \\
\hline Copaifera multijuga Hayne & 0,05 & 0,42 & 15,50 & 0,78 & 0,02 & 0,33 & 0,51 \\
\hline Dinizia excelsa Ducke & 0,03 & 0,23 & 6,58 & 0,33 & 0,07 & 1,33 & 0,63 \\
\hline Diplotropis purpurea (Rich.) Amshoff & 0,01 & 0,10 & 4,46 & 0,22 & 0,00 & 0,06 & 0,13 \\
\hline Diplotropis sp. Benth. & 0,01 & 0,07 & 2,76 & 0,14 & 0,00 & 0,05 & 0,09 \\
\hline Dipteryx odorata (Aubl.) Willd. & 0,14 & 1,15 & 36,09 & 1,81 & 0,07 & 1,30 & 1,42 \\
\hline Enterolobium maximum Ducke & 0,07 & 0,61 & 22,08 & 1,10 & 0,05 & 0,94 & 0,88 \\
\hline Enterolobium schomburgkii (Benth.) Benth. & 0,07 & 0,60 & 21,66 & 1,08 & 0,03 & 0,65 & 0,78 \\
\hline Enterolobium sp. Mart. & 0,06 & 0,52 & 19,96 & 1,00 & 0,02 & 0,48 & 0,67 \\
\hline Eperua schomburgkiana Benth. & 0,03 & 0,27 & 10,40 & 0,52 & 0,01 & 0,18 & 0,32 \\
\hline Hymenaea courbaril $\mathrm{L}$. & 0,18 & 1,54 & 44,37 & 2,22 & 0,12 & 2,34 & 2,03 \\
\hline Hymenaea parvifolia Huber & 0,16 & 1,34 & 41,61 & 2,08 & 0,06 & 1,18 & 1,53 \\
\hline Hymenolobium excelsum Ducke & 0,07 & 0,58 & 21,02 & 1,05 & 0,03 & 0,56 & 0,73 \\
\hline Hymenolobium petraeum Ducke & 0,06 & 0,52 & 20,17 & 1,01 & 0,06 & 1,11 & 0,88 \\
\hline Marmaroxylon racemosum (Ducke) Killip. ex Record. & 0,01 & 0,05 & 2,12 & 0,11 & 0,00 & 0,02 & 0,06 \\
\hline Ormosia paraensis Ducke & 0,02 & 0,14 & 5,94 & 0,30 & 0,01 & 0,10 & 0,18 \\
\hline Ormosia santaremnensis Ducke & 0,01 & 0,10 & 4,03 & 0,20 & 0,00 & 0,07 & 0,12 \\
\hline Parkia multijuga Benth. & 0,18 & 1,51 & 42,46 & 2,12 & 0,06 & 1,21 & 1,62 \\
\hline Parkia pendula (Willd.) Benth. ex Walp. & 0,03 & 0,28 & 10,62 & 0,53 & 0,02 & 0,31 & 0,37 \\
\hline Peltogyne maranhensis Huber ex Ducke & 0,00 & 0,02 & 0,85 & 0,04 & 0,00 & 0,03 & 0,03 \\
\hline Platymiscium duckei Huber & 0,02 & 0,20 & 8,07 & 0,40 & 0,01 & 0,12 & 0,24 \\
\hline Pseudopiptadenia psilostachya (Benth.) G.P.Lewis \& L.Rico & 0,44 & 3,66 & 69,21 & 3,46 & 0,19 & 3,81 & 3,64 \\
\hline Sclerolobium melanocarpum Ducke & 0,20 & 1,71 & 30,57 & 1,53 & 0,07 & 1,36 & 1,53 \\
\hline Sclerolobium melinonii Harms & 0,13 & 1,05 & 23,99 & 1,20 & 0,04 & 0,70 & 0,98 \\
\hline Sclerolobium paraense Huber & 0,03 & 0,24 & 8,70 & 0,44 & 0,01 & 0,18 & 0,28 \\
\hline Stryphnodendron pulcherrimum (Willd.) Hochr. & 0,01 & 0,05 & 1,91 & 0,10 & 0,00 & 0,03 & 0,06 \\
\hline
\end{tabular}


Swartzia ingifolia Ducke

Swartzia sp. Schreb.

Vatairea erythrocarpa (Ducke) Ducke

Vatairea sp. Aubl.

Vataireopsis speciosa Ducke

Zollernia paraensis Huber

Goupiaceae

$\begin{array}{ccccccc}0,01 & 0,05 & 2,34 & 0,12 & 0,00 & 0,03 & 0,07 \\ 0,04 & 0,31 & 11,25 & 0,56 & 0,01 & 0,25 & 0,37 \\ 0,00 & 0,01 & 0,42 & 0,02 & 0,00 & 0,01 & 0,01 \\ 0,07 & 0,59 & 22,29 & 1,12 & 0,02 & 0,49 & 0,73 \\ 0,13 & 1,05 & 29,30 & 1,47 & 0,07 & 1,38 & 1,30 \\ 0,01 & 0,07 & 3,18 & 0,16 & 0,00 & 0,06 & 0,10\end{array}$

$\begin{array}{lllllll}0,35 & 2,91 & 64,12 & 3,21 & 0,16 & 3,09 & 3,07\end{array}$

$\begin{array}{lllllll}0,35 & 2,91 & 64,12 & 3,21 & 0,16 & 3,09 & 3,07\end{array}$

Humiriaceae

Endopleura uchi (Huber) Cuatrec.

Hypericaceae

$\begin{array}{llllllll}0,02 & 0,14 & 4,67 & 0,23 & 0,01 & 0,11 & 0,16\end{array}$

Vismia macrophylla Kunth

$\begin{array}{ccccccc}0,02 & 0,14 & 4,67 & 0,23 & 0,01 & 0,11 & 0,16 \\ \mathbf{0 , 1 5} & \mathbf{1 , 2 5} & \mathbf{2 8 , 4 5} & \mathbf{1 , 4 2} & \mathbf{0 , 0 7} & \mathbf{1 , 3 7} & \mathbf{1 , 3 5}\end{array}$

\section{Lauraceae}

$\begin{array}{lllllll}0,15 & 1,25 & 28,45 & 1,42 & 0,07 & 1,37 & 1,35\end{array}$

Aniba canelilla (Kunth) Mez

$\begin{array}{ccccccc}\mathbf{0 , 3 7} & \mathbf{3 , 0 8} & \mathbf{7 2 , 8 2} & \mathbf{3 , 6 4} & \mathbf{0 , 1 2} & \mathbf{2 , 4 1} & \mathbf{3 , 0 5} \\ 0,02 & 0,14 & 5,31 & 0,27 & 0,00 & 0,08 & 0,16 \\ 0,00 & 0,00 & 0,21 & 0,01 & 0,00 & 0,01 & 0,01 \\ 0,27 & 2,25 & 41,83 & 2,09 & 0,09 & 1,87 & 2,07 \\ 0,02 & 0,16 & 4,88 & 0,24 & 0,00 & 0,09 & 0,16 \\ 0,05 & 0,41 & 15,50 & 0,78 & 0,01 & 0,27 & 0,49 \\ 0,00 & 0,00 & 0,21 & 0,01 & 0,00 & 0,00 & 0,01 \\ 0,00 & 0,02 & 0,85 & 0,04 & 0,00 & 0,02 & 0,03 \\ 0,01 & 0,10 & 4,03 & 0,20 & 0,00 & 0,07 & 0,12\end{array}$

Licaria brasiliensis (Nees) Kosterm.

Mezilaurus itauba (Meisn.) Taub. ex Mez

Mezilaurus lindaviana Schwacke \& Mez

Ocotea baturitensis Vattimo-Gil

Ocotea longifolia Kunth

Ocotea sp. Aubl.

$0,01 \quad 0,10 \quad 4,03$

$\begin{array}{cccccccc}\mathbf{2 , 4 0} & \mathbf{2 0 , 1 5} & \mathbf{2 7 0 , 7 0} & \mathbf{1 3 , 5 4} & \mathbf{1 , 0 2} & \mathbf{2 0 , 2 4} & \mathbf{1 7 , 9 8} \\ 1,12 & 9,35 & 88,32 & 4,42 & 0,49 & 9,82 & 7,86 \\ 0,83 & 6,98 & 82,80 & 4,14 & 0,25 & 5,07 & 5,40 \\ 0,20 & 1,70 & 47,13 & 2,36 & 0,12 & 2,32 & 2,13 \\ 0,22 & 1,86 & 43,31 & 2,17 & 0,14 & 2,78 & 2,27 \\ 0,03 & 0,25 & 9,13 & 0,46 & 0,01 & 0,25 & 0,32\end{array}$

\section{Lecythidaceae}

Couratari guianensis Aubl.

Lecythis lurida (Miers) S.A.Mori

Lecythis pisonis Cambess.

Bertholletia excelsa Humb. \& Bonpl.

$\begin{array}{ccccccc}0,03 & 0,25 & 9,13 & 0,46 & 0,01 & 0,25 & 0,32 \\ \mathbf{0 , 0 9} & \mathbf{0 , 7 6} & \mathbf{2 5 , 4 8} & \mathbf{1 , 2 7} & \mathbf{0 , 0 7} & \mathbf{1 , 3 9} & \mathbf{1 , 1 4}\end{array}$

Lythraceae
Lafoensia glyptocarpa Koehne

Malvaceae

Apeiba macropetala Ducke

$\begin{array}{lllllll}0,09 & 0,76 & 25,48 & 1,27 & 0,07 & 1,39 & 1,14\end{array}$

Ceiba pentandra (L.) Gaertn.

$\begin{array}{lllllll}\mathbf{0 , 0 3} & \mathbf{0 , 2 4} & \mathbf{9 , 1 3} & \mathbf{0 , 4 6} & \mathbf{0 , 0 1} & \mathbf{0 , 2 0} & \mathbf{0 , 3 0} \\ 0,02 & 0,21 & 8,07 & 0,40 & 0,01 & 0,13 & 0,25\end{array}$

\begin{tabular}{|c|c|c|c|c|c|c|c|}
\hline 年 & 管 & , & 我 & , & 型 & , & , \\
\hline Meliaceae & 0,12 & $\mathbf{1 , 0 3}$ & 25,05 & 1,25 & $\mathbf{0 , 0 4}$ & $\mathbf{0 , 8 0}$ & 1,03 \\
\hline Carapa guianensis Aubl. & 0,08 & 0,67 & 11,68 & 0,58 & 0,02 & 0,39 & 0,55 \\
\hline Cedrela fissilis Vell. & 0,00 & 0,01 & 0,42 & 0,02 & 0,00 & 0,01 & 0,01 \\
\hline Cedrela odorata $\mathrm{L}$. & 0,04 & 0,35 & 12,95 & 0,65 & 0,02 & 0,40 & 0,47 \\
\hline Moraceae & $\mathbf{0 , 3 8}$ & 3,15 & 105,94 & 5,30 & 0,17 & 3,37 & 3,94 \\
\hline Bagassa guianensis Aubl. & 0,07 & 0,60 & 19,32 & 0,97 & 0,04 & 0,76 & 0,78 \\
\hline Brosimum acutifolium Huber & 0,05 & 0,39 & 13,59 & 0,68 & 0,02 & 0,33 & 0,47 \\
\hline Brosimum lactescens (S. Moore) C.C.Berg & 0,05 & 0,39 & 12,95 & 0,65 & 0,02 & 0,46 & 0,50 \\
\hline Brosimum parinarioides Ducke & 0,14 & 1,14 & 39,70 & 1,99 & 0,07 & 1,30 & 1,48 \\
\hline Clarisia racemosa Ruiz \& Pav. & 0,07 & 0,63 & 20,38 & 1,02 & 0,03 & 0,54 & 0,73 \\
\hline Myristicaceae & 0,15 & 1,28 & 36,09 & 1,81 & $\mathbf{0 , 0 3}$ & 0,68 & 1,26 \\
\hline
\end{tabular}




\begin{tabular}{lccccccc} 
Virola melinonii (Benoist) A.C. Sm. & 0,15 & 1,28 & 36,09 & 1,81 & 0,03 & 0,68 & 1,26 \\
\hline Olacaceae & $\mathbf{0 , 1 0}$ & $\mathbf{0 , 8 2}$ & $\mathbf{1 1 , 4 6}$ & $\mathbf{0 , 5 7}$ & $\mathbf{0 , 0 2}$ & $\mathbf{0 , 4 5}$ & $\mathbf{0 , 6 2}$ \\
Minquartia guianensis Aubl. & 0,10 & 0,82 & 11,46 & 0,57 & 0,02 & 0,45 & 0,62 \\
\hline Proteaceae & $\mathbf{0 , 0 4}$ & $\mathbf{0 , 3 0}$ & $\mathbf{7 , 6 4}$ & $\mathbf{0 , 3 8}$ & $\mathbf{0 , 0 2}$ & $\mathbf{0 , 4 3}$ & $\mathbf{0 , 3 7}$ \\
Roupala montana Aubl. & 0,02 & 0,19 & 3,61 & 0,18 & 0,02 & 0,35 & 0,24 \\
Euplassa inaequalis (Pohl) Engl. & 0,01 & 0,11 & 4,03 & 0,20 & 0,00 & 0,08 & 0,13 \\
\hline Salicaceae & $\mathbf{0 , 0 3}$ & $\mathbf{0 , 2 9}$ & $\mathbf{1 0 , 1 9}$ & $\mathbf{0 , 5 1}$ & $\mathbf{0 , 0 1}$ & $\mathbf{0 , 1 7}$ & $\mathbf{0 , 3 2}$ \\
Laetia procera (Poepp.) Eichler & 0,03 & 0,29 & 10,19 & 0,51 & 0,01 & 0,17 & 0,32 \\
\hline Sapotaceae & $\mathbf{3 , 2 6}$ & $\mathbf{2 7 , 3 5}$ & $\mathbf{2 4 9 , 8 9}$ & $\mathbf{1 2 , 5 0}$ & $\mathbf{1 , 1 6}$ & $\mathbf{2 3 , 0 3}$ & $\mathbf{2 0 , 9 6}$ \\
Manilkara huberi (Ducke) A. Chev. & 1,74 & 14,57 & 88,75 & 4,44 & 0,72 & 14,37 & 11,12 \\
Manilkara paraensis (Huber) Standl. & 0,27 & 2,25 & 46,07 & 2,30 & 0,07 & 1,32 & 1,96 \\
Micropholis melinoniana Pierre & 0,00 & 0,00 & 0,21 & 0,01 & 0,00 & 0,01 & 0,01 \\
Pouteria bilocularis (H.Winkl.) Baehni & 0,89 & 7,42 & 70,91 & 3,55 & 0,22 & 4,47 & 5,15 \\
Syzygiopsis oppositifolia Ducke & 0,37 & 3,11 & 43,95 & 2,20 & 0,14 & 2,86 & 2,72 \\
\hline Simaroubaceae & $\mathbf{0 , 0 3}$ & $\mathbf{0 , 2 3}$ & $\mathbf{6 , 7 9}$ & $\mathbf{0 , 3 4}$ & $\mathbf{0 , 0 1}$ & $\mathbf{0 , 1 6}$ & $\mathbf{0 , 2 4}$ \\
Simarouba amara Aubl. & 0,03 & 0,23 & 6,79 & 0,34 & 0,01 & 0,16 & 0,24 \\
\hline Vochysiaceae & $\mathbf{0 , 1 9}$ & $\mathbf{1 , 6 2}$ & $\mathbf{4 9 , 0 4}$ & $\mathbf{2 , 4 5}$ & $\mathbf{0 , 1 0}$ & $\mathbf{2 , 0 0}$ & $\mathbf{2 , 0 2}$ \\
\hline Qualea gracilior Pilg. & 0,03 & 0,26 & 9,34 & 0,47 & 0,01 & 0,25 & 0,33 \\
Ruizterania albiflora (Warm.) Marc.-Berti & 0,02 & 0,14 & 5,31 & 0,27 & 0,01 & 0,12 & 0,17 \\
Vochysia maxima Ducke & 0,12 & 1,03 & 26,33 & 1,32 & 0,08 & 1,51 & 1,28 \\
Vochysia sp. Aubl. & 0,02 & 0,20 & 8,07 & 0,40 & 0,01 & 0,11 & 0,24 \\
\hline
\end{tabular}

Total Geral

$\begin{array}{lllllll}11,93 & 100,00 & 1998,94 & 100,00 & 5,02 & 100,00 & 100,00\end{array}$

Onde: DA - densidade absoluta; DR - densidade relativa, FA - frequência absoluta, FR -frequência relativa, DoAdominância absoluta, DoR- dominância relativa e IVI- índice de valor de importância. Fonte: Própria 2019

No entanto, a abundância de indivíduos não segue o mesmo padrão proporcional ao número de espécies, podendo em uma mesma área poucas espécies serem representada por grandes populações (Silva et al., 2014). As espécies mais abundantes na área, em ordem decrescente, foram: Manilkara huberi (Ducke) A. Chev (maçaranduba), Couratari guianensis Aubl (Tauari), Pouteria bilocularis (H.Winkl.) Baehni (goiabão), Lecythis lurida (Miers) S.A.Mori (jarana), Pseudopiptadenia psilostachya (Benth.) G.P.Lewis \& L.Rico (fava timborana) e Syzygiopsis oppositifolia Ducke (Guajará bolacha) (Figura 1).

Em um trabalho feito na Floresta Nacional do Tapajós foi analisado resultados diferentes destacando outras espécies, as que apresentaram maior valor de importância relativo (IVI\%) foram: Pouteria sp. (abiu), Protium apiculatum Swart (breu), Rinorea guianensis (Aubl.) Kuntze (aquariquarana), Eperua bijuga Mart. ex Benth. (cocão) e Eschweilera blanchetiana Miers. (matamatá preto) (Gonçalves e Santos, 2008).

Nesse contexto, a área apresentou um Índice de Shannon - Wiener $\left(\mathrm{H}^{\prime}\right)$ de 3,65 e equabilidade de Pielou (J) de 0,79. De acordo com Knight, 1975 os valores para floresta 
tropical varia de 3,83 a 5,85 sendo que esses valores são considerados elevados para qualquer tipo de vegetação. Além do mais, a riqueza do local está diretamente relacionada com fatores ambientais, latitude, precipitação, nutriente no solo entre outros (Huston, 1980).

Em uma mesma área estuda por Andrade et al., (2008) foram analisados valores de Shannon - Wiener $\left(\mathrm{H}^{\prime}\right)$ de 4,46 e equabilidade de 0,82 evidenciando uma grande diversidade no local. Valores semelhantes foram analisados por Lima et al.,(2018) com índice de Shannon $\left(H^{\prime}\right)$ antes da colheita de madeira foi de 4,10 e 0,85 de equabilidade, enfatizando a diversidade local.

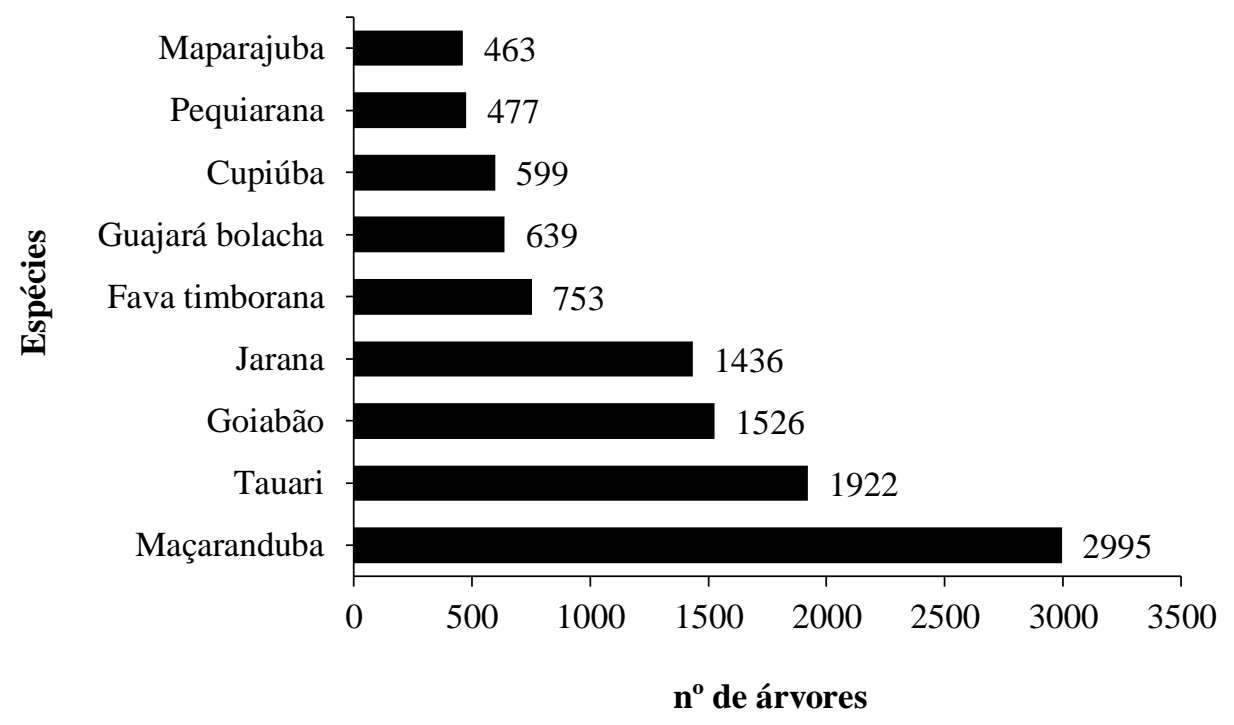

Figura 1. Distribuição das espécies de acordo com numero de arvores na Floresta Nacional do Tapajós. Fonte: autor próprio.

A área em estudo apresentou um crescente número de indivíduos nos centros de classe de diâmetro (CCDAP) de $45 \mathrm{~cm}$ a $95 \mathrm{~cm}$ (Figura 2). De acordo com Andrade (2016) em um estudo feito na Floresta Nacional do Tapajós, a floresta seguiu o padrão de "J- invertido" afirmando que a floresta não sofreu perturbações severas, em sua pesquisa os indivíduos estão mais concentrados nos menores centros de classe, indicando uma floresta jovem, podendo ser explicado pelo fato de não ter sofrido ação antrópica.

No mais, os fatores que podem interferir na distribuição diamétrica das espécies são suas características ecológicas, ciclo de vida, reprodução, ritmo de crescimento e propagação (Cysneiros, 2017). Além disso, explanar sobre a distribuição e composição florística de uma 
área é uma forma de conhecer e aplicar no manejo e conservação da flora nativa da Amazônia (Oliveira et al., 2008).

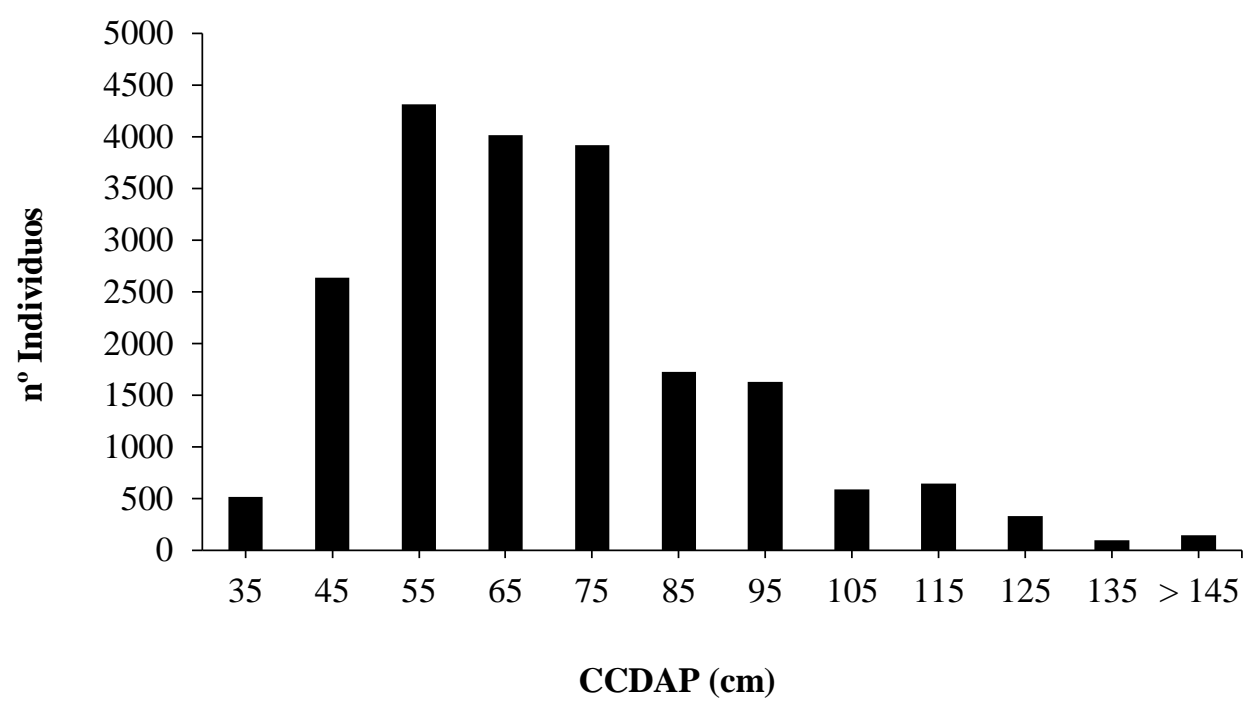

Figura 2. Distribuição diamétrica na Floresta Nacional do Tapajós. Fonte: autor próprio.

Em relação ao volume a área apresentou $69,54 \mathrm{~m}^{3} \cdot \mathrm{ha}^{-1}$, sendo as espécies que apresentaram maiores valores: Manilkara huberi (Ducke) A. Chev (maçaranduba) com 10, 46 $\mathrm{m}^{3}$. ha ${ }^{-1}$, Couratari guianensis Aubl. (tauari) $\left(7,86 \mathrm{~m}^{3} \cdot \mathrm{ha}^{-1}\right)$, Lecythis lurida (Miers) S.A.Mori (jarana) (3,52 $\left.\mathrm{m}^{3} \cdot \mathrm{ha}^{-1}\right)$ e Pouteria bilocularis (H.Winkl.) Baehni (goiabão) (2,86 m³.ha $\left.{ }^{-1}\right)$. Além disso, a área apresentou maior estoque de volume nos diâmetros com DAP $\geq$ que $45 \mathrm{~cm}$ evidenciando maior estoque volumétrico com potencial para exploração de arvores com DAP $\geq 50 \mathrm{~cm}$ (Figura 3).

De acordo com Andrade (2016) na Floresta Nacional do Tapajós as espécies que apresentaram maior volume por hectare em mesma área foram: Manilkara huberi (Ducke) A. Chev $\left(12,12 \mathrm{~m}^{3} \cdot \mathrm{ha}^{-1}\right)$, Pouteria cladanta Sandwith $\left(8,81 \mathrm{~m}^{3} \cdot \mathrm{ha}^{-1}\right)$, Couratari guianensis Aubl $\left(5,82 \mathrm{~m}^{3} \cdot \mathrm{ha}^{-1}\right)$, Alexa grandiflora Ducke $\left(5,75 \mathrm{~m}^{3} \cdot \mathrm{ha}^{-1}\right)$ e Eschweilera grandiflora (Aubl.) Sandwith $\left(5,49 \mathrm{~m}^{3} \cdot \mathrm{ha}^{-1}\right)$. 


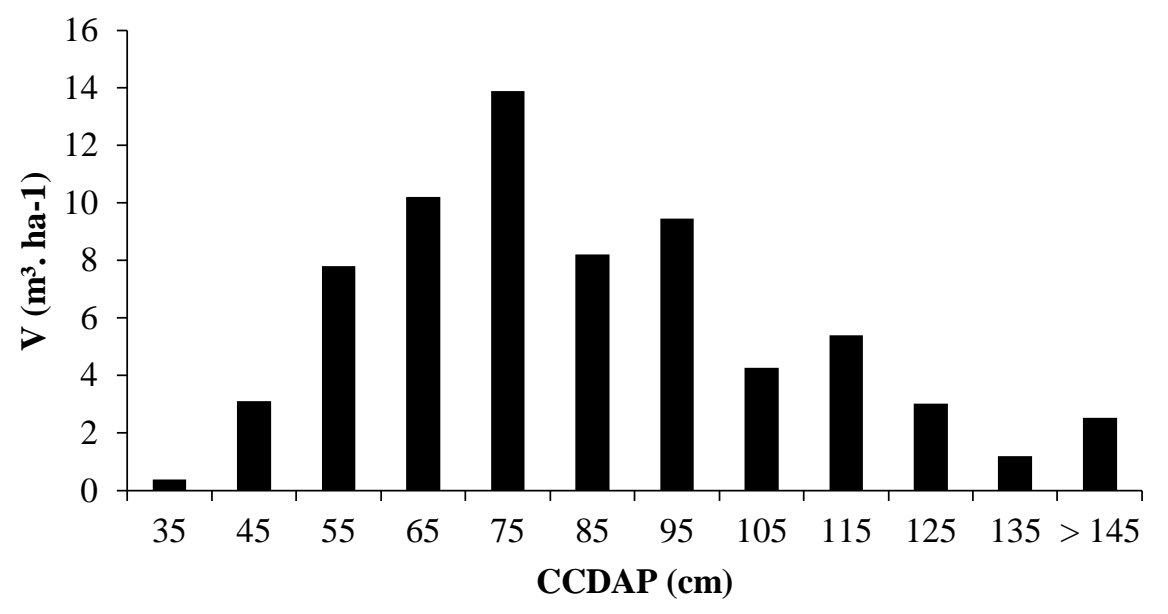

Figura 3. Distribuição volumétrica das espécies na Floresta Nacional do Tapajós.

De acordo com a distribuição espacial pelo índice de Payandeh (Pi) $84 \%$ das espécies apresentam distribuição agregada, 10\% tendência a agrupamento e 6\% aleatória. As espécies que apresentaram maior índice de Payandeh (Pi) e foram classificadas como agregadas são: Manilkara huberi (Ducke) A. Chev (maçaranduba), Couratari guianensis Aubl. (tauari) e Lecythis lurida (Miers) S.A.Mori (jarana). Já em relação à tendência a agrupamento podemos citar Zollernia paraensis Huber (pau-santo) e Swartzia ingifolia Ducke (acapu amarelo). E aleatório as mais evidentes são Protium puncticulatum J.F.Macbr. (breu vermelho) e Micropholis melinoniana Pierre (currupixa).

No entanto, espécies com distribuição espacial com padrão agregada sofrem com a possibilidade de extinção local, pois a área pode sofrer com algum distúrbio afetando todos os indivíduos (Primack e Rodrigues, 2001). A distribuição espacial pode ser afetada por influência do relevo, disponibilidade de nutrientes e água, competição e dispersão de sementes (Capretz et al.,2012). Segundo Nascimento et al.,(2001), as espécies que são classificadas como aleatórias correm em menores valores de indivíduos e podem apresentar problemas de regeneração natural.

\section{Conclusões}

A área apresenta alta diversidade de espécies e bem distribuídas, evidenciando as espécies: Manilkara huberi (Ducke) A. Chev (maçaranduba), Couratari guianensis Aubl (Tauari) e Pouteria bilocularis (H.Winkl.) Baehni (goiabão). Além disso, apresenta estoque potencial para a exploração florestal com espécies concentradas nas maiores classes diamétricas. 


\section{Referências}

CAPRETZ, R. L, BATISTA, J. L. F, SOTOMAYOR, J. F. M, CUNHA, C. R, NICOLETTI, M. F, RODRIGUES, R. R (2012) Padrão espacial de quatro formações florestais do estado de São Paulo, através da Função K de Ripley. Ciência Florestal, 22(3): 551-565. DOI: http://dx.doi.org/10.5902/198050986622.

CAPRETZ, R. L. et al. Padrão espacial de quatro formações florestais do estado de São Paulo, através da função K de Ripley. Ciência Florestal, v. 22, n. 3, p. 551-565, 2012.

CONDÉ, T. M; TONINI, H. Fitossociologia de uma floresta ombrófila densa na Amazônia Setentrional, Roraima, Brasil. Embrapa Agrossilvipastoril-Artigo em periódico indexado (ALICE), 2013.

CURTIS, J.T.; MCINTOSH, R.P. 1951. An upland forest continuum in the prairie-forest border region of Wisconsin. Ecology, 32(3): 476-496.

CUBAS, R. et al. Florística, estrutura e dinâmica em uma floresta ombrófila mista no norte do estado de Santa Catarina. (Dissertação de mestrado) 2011.

CYSNEIROS, V. C. et al. Distribuição diamétrica de espécies da Floresta Ombrófila Densa no Sul do Estado do Rio de Janeiro. Pesquisa Florestal Brasileira, v. 37, n. 89, p. 1-10, 2017.

CYSNEIROS, V. C. et al. Distribuição diamétrica de espécies da Floresta Ombrófila Densa no Sul do Estado do Rio de Janeiro. Pesquisa Florestal Brasileira, v. 37, n. 89, p. 1-10, 2017.

DA SILVA, W. A. S., et al. Composição e diversidade florística em um trecho de floresta de terra firma no sudoeste do estado do Amapá, Amazônia Oriental, Brasil. Biota Amazônia (Biote Amazonie, Biota Amazonia, Amazonian Biota), v. 4, n. 3, p. 31-36, 2014.

DE ALMEIDA LIMA, B. et al. Estrutura e dinâmica florestal sob efeito do manejo madeireiro na FLONA Tapajós. Advances in Forestry Science, v. 5, n. 4, p. 437-443, 2018.

GAMA, J. R. V; BENTES-GAMA, M. M; SCOLFORO, J. R. S (2005) Manejo sustentado para floresta de várzea na Amazônia oriental. Revista Árvore ,29(5): 719-729. DOI: http://dx.doi.org/10.1590/S0100-67622005000500007.

GAMA, J. R. V; SOUZA, A. L; CALEGÁRIO, N; LANA G. C (2007) Fitossociologia de duas fitocenoses de floresta ombrófila aberta no município de Codó, estado do Maranhão. Revista Árvore, 31(3): 465-477. DOI: http://dx.doi.org/10.1590/S0100-67622007000300012.

GONÇALVES, F. G; SANTOS, J. R. Composição florística e estrutura de uma unidade de manejo florestal sustentável na Floresta Nacional do Tapajós, Pará. Acta Amazônica, v. 38, n. 2, p. 229-244, 2008.

HOPKINS, M. J. G. Modelling the known and unknown plant biodiversity of the Amazon Basin. Journal of Biogeography, v. 34, n. 8, p. 1400-1411, 2007. 
HUSTON, M. A. Soil nutrients and tree species richness in Costa Rican forests. Journal of Biogeography, v. 7, p. 147-157, 1980

KNIGHT, D. H. A phytosociological analysis of species-rich tropical forest on Barro Colorado Island, Panama. Ecological Monographs, v. 45, p. 259-28. 1975.

LEHN, C. R; RESENDE, U. M (2007) Estrutura populacional e padrão de distribuição espacial de Cyathea delgadii Sternb. (Cyatheaceae) em uma Floresta Estacional Semidecidual no Brasil Central. Biociências, 13(3-4): 188-195.

MAGURRAN, A. 1988. Ecological diversity and its measurement. Princeton University Press, Princeton, NJ, USA. 179pp.

MUELLER-DOMBOIS, D.; ELLENBERG, G.H. 1974. Aims and methods of vegetation ecology. John Wiley and Sons, Inc., Chichester, England. 547pp.

NASCIMENTO, A. R. T.; LONGHI, S. J.; BRENA, D. A. Estrutura e padrões de distribuição espacial de espécies arbóreas em uma amostra de Floresta Ombrófila Mista em Nova Prata, RS. Ciência Florestal, Santa Maria, v. 11, n.1, p. 105-119, 2001.

OLIVEIRA, A. N. DE; AMARAL, I. L. DO; RAMOS, M. B. P.; NOBRE, A. D.; COUTO, L. B.; SAHDO, R. M. Composição e diversidade florístico-estrutural de um hectare de floresta densa de terra firme na Amazônia Central, Amazonas, Brasil. Acta Amazônica, v. 38, p. 627641. 2008

PRIMACK, R.B.; RODRIGUES, E. Biologia da Conservação. Ed. Planta. 2001, 328p. 